\title{
Evaluación comparativa del nivel de control del asma bronquial en pacientes de 12 a 17 años de edad de EsSalud Arequipa
}

\author{
Arturo Recabarren Lozada ${ }^{1}$, Elvira Parishuaña Calcina ${ }^{2}$, Nadia Martínez Murillo ${ }^{3}$, Grimaneza Esquía Moroco ${ }^{4}$
}

\section{RESUMEN}

Objetivo: Se realizó un estudio observacional, transversal y prospectivo, con el objeto de comparar el nivel de control del asma bronquial en pacientes pediátricos de 12 a 17 años de edad que acuden al Programa de Asma del Hospital III Yanahuara - EsSalud Arequipa con el nivel de control alcanzado en otros centros asistenciales de EsSalud Arequipa, utilizando como instrumento el "Asthma Control Test" (ACT) o "Test de Control del Asma" validado internacionalmente. Materiales y métodos: Se tomó una muestra de 60 pacientes distribuidos en 2 grupos: uno de ellos conformado por 30 pacientes que acuden al Programa de Asma en Pediatría del Hospital Yanahuara y otro por 30 pacientes que acuden a consulta ambulatoria de pediatría de otros centros asistenciales de EsSalud. Para fines del análisis se mantuvo la proporcionalidad en cuanto a sexo y severidad del asma entre ambos grupos.

Resultados: El puntaje alcanzado mediante el ACT fue en promedio de 22,27 en el grupo que asiste al Programa de Asma del Hospital Yanahuara y 20,17 en los que asisten a consulta pediátrica general de otros centros asistenciales. El puntaje más frecuente fue de 25 en el primer grupo y 23 en el otro. Más de la mitad de los pacientes de ambos grupos alcanzaron un puntaje aceptable de control de la enfermedad; sin embargo, el "control total" se logró en mayor proporción en los pacientes que asisten a un programa preventivo (26,67\% versus 6,67\%) y el "pobre control" se observó en mayor proporción en niños evaluados en consulta ambulatoria de pediatría (36,66\% versus 16,66\%).

Conclusiones: La gran mayoría de niños que asisten a un programa preventivo de control del asma logran un control total o aceptable con una diferencia estadísticamente significativa.

Palabras clave: Asma; ACT; programa de asma.

\section{Comparative assessment of bronchial asthma control level in patients aged 12 to 17 years old of EsSalud Arequipa}

\begin{abstract}
Objective: This was an observational, cross-sectional and prospective study to compare the bronchial asthma control level in pediatric patients aged 12 to 17 years old who take part in the Hospital III Yanahuara - EsSalud Arequipa's Asthma Program and have the same control level achieved in other EsSalud Arequipa's health care centers, according to the internationally validated instrument named "Asthma Control Test" (ACT).

Materials and methods: A sample was taken from 60 patients distributed into two groups: one of them consisted of 30 patients who took part in the Hospital Yanahuara Pediatric Unit's Asthma Program and the other one of 30 outpatients from other EsSalud's pediatric health care centers. For the purpose of the analysis, proportionality was maintained with regard to gender and asthma severity in both groups.

Results: The average ACT score was 22.27 in the group who took part in the Hospital III Yanahuara's Asthma Program and 20.17 in the group of outpatients who went to other pediatric health care centers. The most frequent score was 25 for the first group and 23 for the second one. More than half of the patients from both groups achieved an acceptable score in the disease control test. Nevertheless, "total control" was mainly achieved by patients who took part in a preventive program (26.67\% versus 6.67\%) and "poor control" was mainly observed in children assessed in a pediatric outpatient consultation $(36.66 \%$ versus $16.66 \%)$.

Conclusions: The vast majority of children who take part in an asthma control preventive program achieve a total or acceptable asthma control with a statistically significant difference.
\end{abstract}

Keywords: Asthma; ACT; asthma program.

1. Neumólogo - Pediatra, médico asistente de Pediatría del Hospital III Yanahuara - EsSalud, Profesor principal del Departamento de Pediatría - Universidad Nacional de San Agustín de Arequipa.

2. Médico Pediatra, Hospital Nacional Alberto Seguin - EsSalud.

3. Médico Interno, Hospital Regional Honorio Delgado.

4. Licenciada de enfermería, programa de asma del Hospital III Yanahuara. 


\section{INTRODUCCIÓN}

El asma bronquial es una enfermedad crónica frecuente y potencialmente grave que supone una carga considerable para los pacientes, sus familias y la sociedad; provoca síntomas respiratorios, limitación de la actividad y exacerbaciones (crisis o ataques) que en ocasiones requieren asistencia médica urgente y que pueden ser mortales. Por consenso y según Guías Internacionales se define como una enfermedad inflamatoria crónica de vías aéreas bajas, en cuya patogenia intervienen diversas células y mediadores de la inflamación, condicionada en parte por factores genéticos y que cursa con hiperrespuesta bronquial (HRB) y una obstrucción variable del flujo aéreo, total o parcialmente reversible, ya sea por la acción medicamentosa o espontáneamente ${ }^{(1,2)}$. Su incidencia y mortalidad se siguen incrementando y se le considera como el proceso crónico más frecuente en la edad pediátrica. De acuerdo a la Organización Mundial de la Salud, aproximadamente 235 millones de personas sufren de asma en el mundo, cifra que puede ser mayor dado el sub-diagnóstico de la enfermedad; el estudio ISAAC (Internacional Study of Asthma and Allergies in Childhood) muestra prevalencias altas y con tendencia a estabilizarse en países industrializados, mientras que en países en vías de desarrollo parece que la tendencia es al incremento en la prevalencia ${ }^{(3-5)}$.

La prevalencia de síntomas respiratorios sugerentes de asma en los niños en edad escolar ha sido extensamente evaluado en las últimas dos décadas mediante el estudio ISAAC donde se demuestra cifras de prevalencia muy variables a nivel mundial llegando en algunos casos a cifras tan altas como 30\%; en América Latina las cifras son asimismo variables y más de la mitad de países reportan prevalencias mayores al 15\%; en Arequipa utilizando la misma metodología del estudio ISAAC se encontró una prevalencia del 16,29\% en escolares de 13 - 14 años de edad en el año 1996 y una segunda fase de este estudio en el año 2008 reportó una prevalencia de 8,42\% ${ }^{(3,5,6)}$.

Siendo el asma una enfermedad muy común en pediatría y considerando su alta prevalencia en la región, se hace necesario disponer de guías de manejo debidamente documentadas, que permitan orientar a los equipos de salud en el manejo del asma en niños, desde su diagnóstico hasta el tratamiento de los pacientes, de acuerdo con la mejor evidencia disponible y eventualmente con adaptaciones a la realidad de cada país y/o centro hospitalario ${ }^{(2,7-12)}$.

En consideración al impacto social y económico de la enfermedad se hace necesaria la implementación de programas de control y prevención del asma bronquial que persigan como objetivo disminuir la morbimortalidad y mejorar la calidad de vida del paciente asmático. Desde comienzos de la década pasada se ha propuesto disponer de criterios simples (cuestionarios) para precisar si el asma está controlado y que correlacionen de forma aceptable con la función pulmonar ${ }^{(13,14)}$. Por lo que es importante contar con instrumentos sencillos y de fácil aplicación que permitan tener una apreciación del nivel del control del asma obtenida por un paciente en tratamiento, así un grupo de expertos han desarrollado un instrumento que cuantifica el control del asma con o sin el uso de pruebas de función pulmonar, como es el cuestionario "Asthma Control Test" (ACT) o "Test de Control del Asma" (15).

Dada la importante prevalencia del asma bronquial en nuestro medio y la existencia de un programa preventivo en el Hospital III Yanahuara - EsSalud, se decidió desarrollar el presente trabajo de investigación para evaluar y comparar el nivel de control de asma alcanzado en pediatría y en diferentes establecimientos de EsSalud - Arequipa.

Se plantea como hipótesis que: “Los pacientes de 12 a 17 años de edad que acuden a un programa preventivo de control de asma tienen un mejor nivel de control de su enfermedad".

Los objetivos del presente estudio fueron:

1. Evaluar el nivel de control del asma en niños asmáticos de 12 a 17 años de edad mediante el ACT.

2. Comparar el nivel de control del asma de los pacientes que acuden a un programa preventivo versus pacientes que acuden sólo a consulta ambulatoria de pediatría.

\section{MATERIALES Y MÉTODOS}

Según Altman D, el presente estudio es de tipo observacional, transversal y prospectivo. La población estuvo constituida por pacientes con el diagnóstico de asma bronquial, con el requisito de haber presentado 3 o más episodios de sibilancias corroboradas por un médico pediatra en el último año y/o haber sido evaluados por el neumólogo pediatra en el programa de asma del Hospital III Yanahuara.

El tamaño de la muestra se realizó por conveniencia y la selección se hizo de manera probabilística manteniendo la proporcionalidad en cuanto a sexo y severidad de la enfermedad; se trabajó con una muestra de 60 niños divididos en 2 grupos, uno de ellos (30 niños) que acuden al programa preventivo de asma bronquial del Hospital III Yanahuara EsSalud y otro (30 niños) que acuden a consulta ambulatoria de pediatría de otros centros asistenciales de EsSalud y que cumplieron con los siguientes criterios.

\section{Criterios de inclusión}

1. Niños de 12 a 17 años 11 meses con el diagnóstico clínico de asma bronquial.

2. Niños de ambos sexos.

3. Niños que se reciban tratamiento para el asma indicado por el pediatra o neumólogo - pediatra, en los últimos 12 meses. 


\section{Criterios de exclusión}

1. Niños en el rango de edades y sexo antes descritos que no sean ubicables hasta en tres oportunidades en su domicilio.

El instrumento utilizado fue el "Test de Control de Asma" o ACT el mismo que consta de 5 interrogantes con 5 alternativas de respuesta que se cuantifican con una puntuación que va de 1 a 5 , calificando con puntajes mayores las respuestas que sugieren un buen control o un control total de la enfermedad y puntajes menores las que sugieren un control inadecuado de la enfermedad; al niño se le pide que haga un círculo alrededor del puntaje adecuado para su condición y que por cada pregunta escriba el número respectivo en el casillero de la derecha.

Las 5 preguntas que se evalúan son:

a) Durante las últimas 4 semanas, ¿con qué frecuencia su asma le impidió completar sus tareas habituales en el trabajo, los estudios o el hogar?
1. Siempre
2. Casi siempre
3. Algunas veces
4. Pocas veces
5. Nunca

b) Durante las últimas 4 semanas, ¿con qué frecuencia sintió falta de aire?

1. Más de una vez por día

2. 1 vez por día

3. De 3 a 6 veces por semana

4. 1 ó 2 veces por semana

5. Nunca

c) Durante las últimas 4 semanas, ¿con que frecuencia sus síntomas de asma (silbidos en el pecho, tos, falta de aire, opresión en el pecho) lo despertaron durante la noche o más temprano que de costumbre por la mañana?
1. 4 ó más noches por semana
2. 2 ó 3 noches por semana
3. 1 vez por semana
4. 1 ó 2 veces
5. Nunca

d) Durante las últimas 4 semanas, ¿con que frecuencia usó su inhalador de efecto inmediato o se hizo nebulizaciones (por ejemplo, salbutamol)?

1. 3 ó más veces por día

2. 1 ó 2 veces por día

3. 2 ó 3 veces por semana

4. 1 vez por semana o menos

5. Nunca e) ¿Cómo calificaría el control de su asma durante las últimas 4 semanas?

1. Por nada controlada

2. Mal controlada

3. Algo controlada

4. Bien controlada

5. Totalmente controlada

La calificación final considera de la siguiente manera los distintos niveles de control:

- Control Total $=25$ puntos

- Control Aceptable = 20 - 24 puntos

- Control Pobre $=<20$ puntos

Para la obtención y evaluación de las respuestas del ACT se realizaron visitas domiciliarias hasta en 2 ocasiones, procediendo a registrar las mismas previo consentimiento de los padres o tutores de los niños; a cada niño se le dio un tiempo de 5 a 10 minutos para responder el cuestionario respectivo. En cada grupo y en forma proporcional se evaluó a 17 niños con asma persistente leve, 12 niños con asma persistente moderado y 1 niño con asma persistente severo.

Los datos obtenidos fueron vaciados a una base de datos Excel, aplicando posteriormente estadística descriptiva calculando la media aritmética, mediana y DS para cada grupo; para la comparación de ambos grupos se utilizó la prueba U de Mann Whitney por tratarse de datos ordinales.

\section{RESULTADOS}

Se estudiaron a 60 pacientes de 12 a 17 años de edad, distribuidos en dos grupos de 30 niños respectivamente; uno de los grupos tuvo un control regular de su enfermedad a través de un programa preventivo de asma como es el programa de control de asma en pediatría del Hospital III Yanahuara, donde son evaluados por el neumólogo pediatra del Hospital III Yanahuara, en forma periódica y con citas programadas cada 3 meses, además de recibir educación adicional a cargo de la enfermera del programa cada vez que los padres del niño recogen su medicación, que según el caso es cada 30 a 45 días; y el otro grupo de estudio se controló en consulta ambulatoria en pediatría para la evaluación de su enfermedad, en ambos casos por un período no menor de 12 meses.

La Tabla 1 muestra la distribución de los pacientes en ambos grupos de estudio, divididos por edad, sexo y severidad de la enfermedad; se aprecia que la distribución de los pacientes por edad guarda proporcionalidad entre ambos grupos al igual que la distribución por sexo y severidad del asma, lo que permite la comparación óptima de ambos grupos. 
Tabla 1. Distribución de los pacientes de ambos grupos según edad, sexo y severidad del asma

\begin{tabular}{|c|c|c|c|c|}
\hline \multirow{2}{*}{ Edad en años } & \multicolumn{2}{|c|}{ Programa de asma } & \multicolumn{2}{|c|}{ Consulta externa pediatría } \\
\hline & $\mathbf{N}^{\circ}$ & $\%$ & $\mathbf{N}^{\circ}$ & $\%$ \\
\hline 12 & 6 & 20,00 & 5 & 16,67 \\
\hline 13 & 9 & 30,00 & 9 & 30,00 \\
\hline 14 & 5 & 16,66 & 7 & 23,33 \\
\hline 15 & 6 & 20,00 & 6 & 20,00 \\
\hline 16 & 2 & 6,67 & 3 & 10,00 \\
\hline 17 & 2 & 6,67 & 0 & 0,00 \\
\hline \multicolumn{5}{|l|}{ Sexo } \\
\hline Masculino & 15 & 50,00 & 15 & 50,00 \\
\hline $\begin{array}{l}\text { Femenino } \\
\text { Severidad del } \\
\text { asma persistente }\end{array}$ & 15 & 50,00 & 15 & 50,00 \\
\hline Leve & 17 & 56,67 & 17 & 56,67 \\
\hline Moderado & 12 & 40,00 & 12 & 40,00 \\
\hline Severo & 1 & 3,33 & 1 & 3,33 \\
\hline Total & \multicolumn{2}{|c|}{30} & \multicolumn{2}{|c|}{30} \\
\hline
\end{tabular}

La tabla 2 muestra las medidas estadísticas resumen en forma comparativa entre ambos grupos; se aprecia que el puntaje promedio es mayor en el grupo de niños que acuden a un programa preventivo al igual que el puntaje que se repite con mayor frecuencia que es 25 (control total) en este mismo grupo.

Tabla 2. Puntaje alcanzado del Test de control del asma, según diversas medidas

$\begin{array}{lcr} & \text { Medida } & \text { Consulta externa pediatría } \\ \text { Media aritmética } & 22,27 & 20,17 \\ \text { Mediana } & 23 & 20 \\ \text { Moda } & 25 & 23 \\ \text { Desviación estándar } & 2,55 & 3,22 \\ \text { Mínimo } & 17 & 13 \\ \text { Máximo } & 25 & 25 \\ \text { Total } & 30 & 30\end{array}$

La Tabla 3 y Figura 1 muestran la calificación cualitativa final obtenida entre ambos grupos y evaluada mediante el ACT; se aprecia nuevamente que los niños que acuden a un programa preventivo logran los puntajes mejores logrando con mayor frecuencia un "control total" o "aceptable" de la enfermedad. 
Tabla 3. Comparación del Test del control del asma en ambos grupos según nivel de control del asma bronquial

\begin{tabular}{lrcrr}
\multicolumn{1}{c}{$\begin{array}{c}\text { Nivel de Control } \\
\text { del asma }\end{array}$} & \multicolumn{2}{c}{ Programa de asma } & \multicolumn{2}{c}{ Consulta externa pediatría } \\
Total & $\mathbf{N}^{\circ}$ & $\%$ & $\mathbf{N}^{\circ}$ & 2 \\
Aceptable & 8 & 26,67 & 17 & 6,67 \\
Pobre & 17 & 56,67 & 11 & 36,67 \\
Total & 5 & 16,66 & 30 & 36,66 \\
& 30 & 100,00 & & 100,00
\end{tabular}

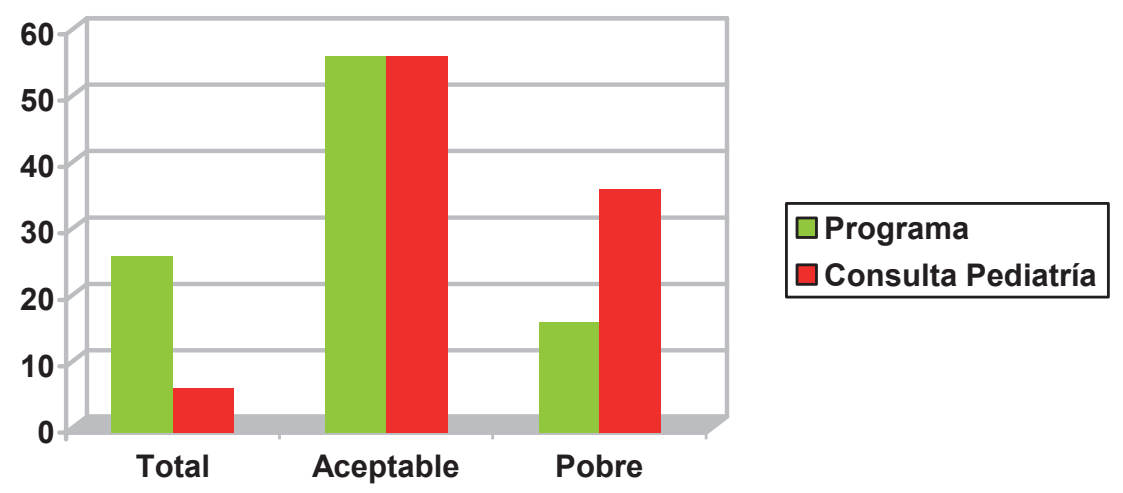

$U=309 \quad Z=-2,33 \quad P=<0,01$

Figura 1. Comparación del Test del control del asma en ambos grupos según nivel de control del asma bronquial

\section{DISCUSIÓN}

El Asma bronquial es la enfermedad crónica más común en pediatría y su prevalencia tiene aún tendencia al incremento, a pesar de existir una mejor comprensión de su fisiopatología y de conocer que siendo una enfermedad inflamatoria crónica, la terapia debe abordar este aspecto en forma sostenida, única manera de lograr el control de la enfermedad ${ }^{(1,5,16)}$.

Por su condición de cronicidad, el asma requiere generalmente asistencia médica regular en el tiempo, y por ello es importante diseñar programas específicos para su manejo correcto que impliquen la mejor adherencia posible al uso continuo de medicación antinflamatoria vale decir al uso de Corticoides Inhalados (ICS) por parte del paciente y la educación continua que permita llevar una vida normal del niño y enseñar al mismo y a los padres a evitar los posibles factores desencadenantes ${ }^{(8,10,11)}$. En el Programa de Asma del Hospital III Yanahuara - Arequipa, se realiza la atención del niño con asma mediante la participación de un neumólogo pediatra y una licenciada en enfermeria capacitada en la educación integral del paciente y su familia; el diagnóstico de asma se realiza mediante valoración clínica a la que se agrega al ingreso al programa, pruebas de función pulmonar como espirometría y/o prueba de provocación bronquial con el ejercicio que permiten catalogar al niño con asma en determinado grado de severidad, iniciando luego un tratamiento controlador a largo plazo; la mayoría de los niños recibe Beclometasona como ICS ya que es el medicamento que se dispone usualmente en la institución, aunque en algunos niños que requieren dosis mayores de ICS se emplea Fluticasona; Las dosis de Beclometasona son de: 100 - 300 ugr/día para el asma persistente leve, de 300 a 600 ugr/día para el asma persistente moderado y > 600 ugr/día para el asma persistente severo, dosis que se van disminuyendo en forma progresiva y en un período no menor de 6 meses una vez logrado el control de la enfermedad; es importante mencionar que las dosis altas se emplean excepcionalmente ya que de todos los niños inscritos en el Programa y con control regular no más del $2 \%$ tienen asma severo.

Como se aprecia la valoración del control del asma implica aspectos clínicos evaluados por el médico tratante, adicionalmente valoración del grado de inflamación y pruebas funcionales que ayuden a precisar el diagnóstico y catalogación de la severidad, pero que 
lamentablemente no están ampliamente disponibles en todos los establecimientos de salud de nuestro país ${ }^{(17-20)}$; la modalidad del cuestionario es una alternativa de valoración y su utilidad para estudiar enfermedades pulmonares ya fue validada por pruebas de función pulmonar ${ }^{(13,15,21)}$.

En el presente estudio se utiliza como instrumento para valorar el control del asma al cuestionario: "Asthma Control Test" (ACT) o "Test de Control del Asma" que es un cuestionario desarrollado por la American Academy of Allergy Asthma \& Inmunology y que ha sido validado internacionalmente; el ACT tiene 2 versiones una para niños de 12 a más años y adultos cuyo cuestionario es respondido por el propio paciente y otra para niños de 4 a 11 años cuyo cuestionario se responde por el niño y sus padres ${ }^{(15,21-23)}$. En base a este cuestionario se evalúa el control del Asma bronquial en niños de 12 a 17 años de edad del programa preventivo de asma del Hospital III Yanahuara y se compara con el nivel de control alcanzado en niños de otros Centros asistenciales de EsSalud donde no tienen un programa de control de asma por lo que la evaluación y seguimiento de estos niños se realiza en consulta ambulatoria de pediatría.

En cuanto a la distribución de los pacientes por edad se aprecia una distribución homogénea entre ambos grupos con un predominio de niños de 13 y 14 años de edad (alrededor del $50 \%$ de los casos); no existe diferencia en cuanto al sexo, siendo la proporción similar en niños de un programa preventivo y niños de consulta ambulatoria (Tabla 1). En cuanto a la severidad de la enfermedad se tomó asimismo una distribución proporcional entre ambos grupos, presentando 17 niños asma persistente leve, 12 niños asma persistente moderado y uno asma persistente severo por cada grupo de estudio.

La tabla 2 muestra el puntaje final del ACT obtenido en ambos grupos, encontrando que los niños que acuden a un programa preventivo obtienen puntajes superiores con un promedio de 22,27; mientras que los niños asmáticos que se controlan en consulta externa de pediatría tienen un puntaje menor 20,17. Esto se reafirma y deja notar más cuando se revisa el puntaje que se repite con mayor frecuencia, siendo 25 en los niños del programa de asma valer decir un "control total" y 23 en los niños en consulta ambulatoria pediátrica vale decir un "control aceptable". Asimismo el puntaje mínimo encontrado en los niños del programa de control de asma es mayor que en los niños que no acuden a un programa preventivo (17 versus 13 ); todas estas diferencias son estadísticamente significativas.

Evaluar los resultados mediante la encuesta ACT que es simple de aplicar nos refleja que este instrumento puede ser útil a los médicos clínicos y pediatras que estén interesados en la determinación del nivel de control de la enfermedad dentro de su práctica clínica y a los investigadores que intentan determinar el nivel de control del asma dentro de una población con o sin el uso de pruebas de función pulmonar; los instrumentos tales como el ACT desempeñan un papel importante en esfuerzos en curso, y futuras evaluaciones para refinar las pautas de tratamiento del asma; por otro lado la experiencia con el uso de este instrumento ha sido satisfactoria y su validación y uso se reporta en diversos países latinoamericanos, además de existir encuestas similares que se han empleado en otras partes del mundo ${ }^{(21-23)}$.

Al evaluar las categorías que alcanzaron los grupos de estudio de acuerdo al puntaje del test (Tabla 3 y Figura 1), vemos que en algo más de la mitad y en ambos grupos tuvieron un control considerado como "aceptable". Las diferencias radican en que el grupo de pacientes que acuden a un programa preventivo se encontró que más de la cuarta parte $(26,67 \%)$ refirieron a través del test tener un "control total" del asma mientras que en el grupo de consulta pediátrica la cifra es significativamente menor $(6,67 \%)$; en otras palabras por cada niño que logra un control total de la enfermedad en ausencia de un programa preventivo existen 4 niños con control total si se les sigue en forma regular en el programa de asma. Asimismo es importante resaltar que por cada paciente con "control pobre" de los que asisten a un programa preventivo existen dos con "control pobre" de los que sólo se atienden en consulta ambulatoria de pediatría (16,66 versus $36,66 \%$ respectivamente), todos estos registros son estadísticamente significativos.

Siendo el asma una enfermedad crónica, la educación sobre la enfermedad juega un rol vital en el manejo de la misma ya que además de prescribir la terapia antiinflamatoria apropiada es indispensable asegurarnos que el niño y sus padres comprendan la forma de acción de la medicación empleada, la necesidad de su uso sostenido, la forma correcta de uso de los inhaladores y el empleo del espaciador o aerocámara, además deben comprender cómo evitar los factores desencadenantes y agravadores y cómo manejar en forma inicial una exacerbación, todo ello llevará a un control óptimo de la enfermedad y a lograr la adherencia a la medicación indicada ${ }^{(2,7-9)}$. Muchas veces la intervención aislada del Médico puede ser insuficiente para abordar todos estos aspectos ya que el tiempo que se le asigna para la atención de niños por hora y las "metas" que debe alcanzar impiden abordar integralmente estos aspectos educativos; en cambio, cuando se instaura un programa preventivo es más factible lograr estos objetivos por la participación de diversos especialistas y la atención más individualizada por parte de enfermería, que puede apoyar en la educación de los padres y del propio niño además de colaborar en la realización de pruebas complementarias en estos pacientes ${ }^{(17-19,24)}$.

El asma inducido por ejercicio (EIB) es un evento común en el asma y lo presentan aproximadamente un $65-70 \%$ de niños con esta enfermedad, el ACT ha mostrado una correlación buena cuando se valora esta variable, y el cuestionario ACT predijo correctamente la ausencia de EIB, si el puntaje obtenido fue $>19^{(25)}$. En un estudio local reciente, se aprecia que cuando se realiza en forma sostenida el tratamiento de un niño asmático con la medicación controladora (corticoides inhalados) y se correlaciona la mejoría clínica con algún marcador inflamatorio, como por ejemplo nitritos en el esputo inducido, se observa de manera significativa que el marcador inflamatorio 
disminuye sustancialmente luego del uso continuo del corticoide, aspecto que es más factible de lograr en un programa preventivo donde se remarca que el uso del inhalador (ICS) debe ser continuo ${ }^{(1,2,24,26)}$. Una ventaja adicional del ACT, es que el mismo se puede responder vía Web, versión que ya ha sido validada y que muestra un costo - efectividad superior a otras formas de monitorización como por ejemplo la medición de FENO; la posibilidad de responder online el cuestionario es interesante, ya que la página web respectiva (www.asthmacontroltest. com) tiene un sponsor farmacéutico que en forma gratuita permite acceder al cuestionario (disponible en diversos idiomas) responderlo como estrategia de control del asma por ejemplo 1 vez al mes, y tener en base al puntaje final una apreciación del nivel de control con sugerencias de acción que eventualmente pueden sugerir la visita al médico tratante para modificaciones en el tratamiento y alcanzar el "control total" de la enfermedad ${ }^{(20,27)}$.

Es conocido que la exposición a alérgenos o humo de cigarrillo dentro del hogar, alérgenos externos y polución ambiental pueden exacerbar los síntomas de asma y eventualmente provocar mayores consultas en emergencia y hospitalizaciones ${ }^{(28-31)}$. Por lo que la educación del paciente con asma se considera uno de los pilares del tratamiento y es un proceso continuo cuyo objetivo es proporcionar la información necesaria y promover las conductas adecuadas para poder aplicar correctamente el plan de tratamiento, la comunicación deberá ser fluida y de mutua confianza entre el personal de salud, el niño con asma y sus padres; por otro lado la identificación de los desencadenantes medioambientales sean estos alérgenos o irritantes físicos o químicos que inducen la inflamación bronquial es importante y evitarlos puede contribuir en forma clara a una reducción de los síntomas ${ }^{(2,8-10)}$.

Existen grupos de niños que requieren un manejo diferenciado como son el adolescente y el niño con sobrepeso - obesidad; el primero tiene tendencia a "negar" la enfermedad y por consiguiente abandonar el tratamiento y el segundo tiene por su propia condición nutricional menor control de la enfermedad con incremento de síntomas diurnos y nocturnos y función pulmonar más comprometida con menores valores del PEF respecto a niños asmáticos eutróficos ${ }^{(32-34)}$. Finalmente es importante mencionar que si bien el ACT es un instrumento valioso en el manejo del niño asmático, no necesariamente existe una correlación entre la apreciación que tiene el paciente y/o sus padres de su enfermedad, contrastada con la opinión del pediatra o neumólogo pediatra, hecho que se describió con el cuestionario para niños de 4 a 11 años de edad ${ }^{(35)}$.

Como se aprecia, los objetivos que se deben alcanzar son múltiples y para lograrlos se requiere la participación de un equipo multidisciplinario que idealmente debe incluir al médico, enfermera, fisioterapeuta, asistenta social y nutricionista; obviamente que lograr este tipo de abordaje integral requiere compromiso de parte del médico general o pediatra en el control y educación continua del niño asmático y/o la instauración de programas preventivos, en este caso se tiene ya la experiencia por más de 15 años en el programa preventivo de asma en pediatría del Hospital III Yanahuara de Arequipa ${ }^{(6,17,19,32)}$.

En conclusión, en el presente estudio se demuestra que la gran mayoría de niños de 12 a 17 años de edad que asisten a un programa preventivo de control del asma, logran un nivel de control del asma "total" o "aceptable" con diferencia estadísticamente significativa respecto a los controles.

\section{REFERENCIAS BIBLIOGRÁFICAS}

1. Global Initiative for Asthma (GINA), guía de bolsillo para el manejo y la prevención del asma. 2016; 1 - 35.

2. Guía Española para el manejo del Asma (GEMA). Comité Ejecutivo de la GEMA. 2015; 1 - 164.

3. Mallol J, Solé D, Asher I, Stein R, Soto-Quiroz M. Prevalence of asthma symptoms in Latin America: the International Study of Asthma and Allergies in Childhood (ISAAC). Pediatr Pulmonol. 2000; 30(6): 439 - 444.

4. Akimbami L, Simon A, Rossen L. Changing trends in asthma prevalence among children. Pediatrics. 2016; 137(1):2015354.

5. Forno E, Gogna M, Cepeda A, Yáñez A, Solé D, Cooper P, et al. Asthma in Latin America. Thorax. 2015; 70 (9): 898 - 905.

6. Recabarren A, Cusirramos D, Gutiérrez V, Andrade P, Chiarella P. Prevalencia y severidad del asma bronquial en niños de 13 - 14 años de la ciudad de Arequipa y su variación en 12 años (1996 - 2008). Neumología Pediátrica 2010; 5(Supl 1): 32.

7. Guía de práctica clínica sobre asma infantil. Ministerio de Sanidad, servicios sociales e igualdad. Gobierno Vasco. 2015; $1-162$.

8. Gobierno de Chile: Guía clínica AUGE, Asma bronquial moderada y grave en menores de 15 años. Serie guías clínicas MINSAL. 2011; 1 - 48.

9. Giubergia V, Balanzat A, Teper A, Vidaurreta S. Guía de diagnóstico y tratamiento: asma bronquiial en niños > 5 años. Arch Argen Pediatr. 2016; 114 (6): 595 - 6

10. Gobierno Federal de México: Diagnóstico y tratamiento del asma en menores de 18 años de edad. Guía de práctica clínica GPC. 2013; 1 - 20.

11. Carvalho C, Baldi B, Viana C, Caruso P. Diretrizes da Sociedade Brasileira de Pneumología e Tisiología para o Manejo da Asma. Jornal Brasileiro de Pneumologia. 2012; 38(supl1):1 - 46.

12. Rodrigo G, Plaza V, Bardagi S, Castro-Rodríguez J, Damiá A, Liña S, et al. ALAT Guía Alerta 2. América Latina y España: Recomendaciones para la prevención y el tratamiento de la exacerbación asmática. Arch Bronconeumol. 2010; 46 (Supl 7): 1 - 20.

13. Boulet $L$, Boulet V, Milot J. How should we quantify asthma control ? Chest. 2002; 122(6): 2217 - 23.

14. Bateman E, Boushey H, Bousquet J, Busse W, Clark T, Pauwels $\mathrm{R}$, et al. Can guideline-defined asthma control be achieved? The Gaining Optimal Asthma ControL Study. Am J Respir Crit Care Med. 2004; 170(8): 836 - 44.

15. Nathan R, Sorkness C, Kosinski M, Schatz M. Development of the asthma control test: a survey for assessing asthma control. J All Clin Inmunol 2004;113(1):59- 65.

16. Fuhlbrigge A, Adams R, Guilbert T, Grant E, Lozano P, Janson $P$, et al. The burden of asthma in the United States: level and distribution are dependent on interpretation of the National asthma education and prevention program guidelines. Am J Respir Crit Care Med 2002; 166(8): 1044 - 9. 
17. Recabarren A, Apaza C, Castro-Rodríguez J. Nitrites in induced sputum as a simple and cheap non-invasive marker of airway inflammation for asthmatic schoolchildren. Pediatr Allergy Immunol. 2008; 19(5): 433 - 7.

18. Brouwer A, Roorda R, Brand P. Comparison between Peak Expiratory Flow and FEV1 measurements on a home spirometer and on a pneumotachograph in children with asthma. Pediatr Pulmonol. 2007; 42(9): 813 - 8.

19. Lazo-Velásquez J, Lozada A, Cruz H. Evaluation of severity of bronchial asthma through an exercise bronchial challenge. Pediatr Pulmonol. 2005; 40(5):457- 63.

20. Beerthuizen T, Voored-van S, van den Hout W, VaessenVerberne A, Brackel H, Landstra A, et al. Cost-effectiveness of FENO-based and web-based monitoring in paediatric asthma management: a randomised controlled trial. Thorax. 2016; 71(7): 607 - 13.

21. Voorend-van Bergen, Vaessen V, Jongste JC, Pijnemburg M. Asthma control questionnaires in the management of asthma in children: a review. Ped Pulmonol 2015; 50(2): 202-8.

22. Oliveira S, Sarria E, Roncada C, Stein R, Pitrez M, Matiello R. Validation of the Brazilian version of the childhood asthma control test (ACT). Ped Pulmonol. 2016; 51(4): 358 - 63.

23. Bussi L, Sly P, Restuccia S, LLancamán L. Validation of a schoolbased written questionnaire for asthma case identification in Argentina. Ped Pulmonol. 2012; 47(1): 1 - 7.

24. Castro-Rodríguez J, Molina R, Cáceres $M$, Recabarren A. Correlation between Nitrites in induced sputum and asthma symptoms in asthmatic schoolchildren. Ped Pulmonol. 2014; 49(3): 214 - 20.

25. Chinellato I, Piazza M, Sandri M, Cardinali F, Peroni G, Boner $A$, et al. Evaluation of association between exercise-induced bronchoconstriction and childhood asthma control test (ACT) questionnaire scores in children. Pediatr Pulmono.l 2012; 47(3): $226-32$.

26. Pedersen S, Hurd S, Lemanske R, Becker A, Zar H, Sly P, et al. Global strategy for the diagnosis and mangement of asthma in children 5 years and younger. Pediatr Pulmonol. 2011; 46(1): 1 - 17.

27. Koolen B, Pignenburg M, Brackel $H$, Landstra A, Van den Bergh $N$, Merkus $P$, et al. Validation of a web-based versión of the asthma control test and chidhood asthma control test. Pediatr Pulmonol. 2011; 46(10): 941 - 8.

28. Murray C, Foden P, Sumner H, Shepley E, Custovic A, Simpson A. Preventing severe asthma exacerbations in children: a randomised trial of mite impermeable bedcovers. American Journal Respir Critical Care Medicine. 2017
29. Farber H, Batsell R, Silveira E, Calhoun R, Giardino A. The impact of tobacco smoke exposure on childhood asthma in a Medicaid managed Care Plan.Chest 2016; 149 (3): 721 - 8.

30. Chen C, Chao H, Chang C, Chen B, Guo Y. Current asthma in schoolchildren is related to fungal spores in classrooms. Chest. 2014; 146(1): 123 - 34.

31. Barrat B, Quint J. Asthma hospitalizations and air pollution. Thorax. 2016; 71(12): 1076 - 7.

32. Recabarren A, Portugal K, Gutiérrez J. Comparación de las características clínicas del asma bronquial entre niños con sobrepeso/obesidad y niños eutróficos inscritos en el programa de asma bronquial del Hospital III Yanahuara EsSalud Arequipa. Diagnóstico 2003; 42(2): 60 - 7.

33. Bitsko M, Everhart R, Rubin B. The adolescent with asthma. Paediatr Respir Rev. 2013; 15(2): 146 - 53.

34. Jensen M, Collins C, Gibson P, Wood L. The obesity phenotype in children with asthma. Paediatr Respir Rev. 2011; 12(3): 152 $-9$.

35. Shefer G, Donchin M, Manor O, Levy-Hervroni R, Schechter A, Cohen $\mathrm{H}$, et al. Disparities in assesments of asthma control between children, parents and physicians. Pediatr Pulmonol. 2014; 49(10): 943 - 51.

\section{Fuentes de financiamiento:}

Este artículo ha sido financiado por los autores.

\section{Conflictos de interés:}

Los autores declaran no tener ningún conflicto de interés.

\section{Correspondencia:}

Arturo Recabarren Lozada

Dirección: Filomena 102. Pueblo Viejo, Cerro Colorado.

Teléfono: 959654097

Correo electrónico: arturorecabarren@gmail.com

Recibido: 28 de febrero de 2017 Aprobado: 31 de marzo de 2017 